\title{
Effect Of Source Tea On The Normal Mice's Gastrointestinal Function Wang Ting ${ }^{1}$, Li Yan¹, Wang Tan¹, Wang Zuhong ${ }^{2}$, Bai Ming ${ }^{1}$ and Miao Mingsan ${ }^{1, *}$ \\ ${ }^{1} 1$ Henan University of Traditional Chinese Medicine, Zhengzhou \\ ${ }^{2}$ Fusen pharmaceutical, Nanyang \\ *corresponding author: Miao Mingsan
}

Keywords: Fusen herbal tea ;gastrointestinal function

\begin{abstract}
Effect of observation Fusen herbal tea in normal mice methods.: given to mice for 17 days, daily 2 times respectively in drinking high dose Fusen herbal tea, small dose Fusen herbal tea, Wanglaoji large dose of herbal tea, small dose of Wanglaoji herbal tea, herbal tea replace normal mice drinking water; control group received normal drinking water. In 17 days, the mice were sacrificed and the body weight of mice, drinking water and feed amount and bio electrical changes. Results: group and blank ratio, large and small dose Fusen herbal tea in mice body weight, drinking water and feed amount and bio electricity situation no greater impact. Conclusion: Fusen herbal tea on mice body weight, drinking water and feed amount and no obvious adverse effects.
\end{abstract}

\section{Introduction}

From honeysuckle, licorice, immortality, dried ginger, chrysanthemum, radix puerariae, hawthorn, Fusen herbal tea yam, the herbs are containing components cool, people's gastrointestinal function for the cold sensitive components, improper use can cause gastrointestinal weakness aggravating [1], so for the observed long-term drinking a lot of Fusen herbal tea in normal mice gastrointestinal effect.

\section{Experimental Materials}

Experimental Drugs. Fusen herbal tea: from honeysuckle, chrysanthemum, grass jelly, licorice, dried ginger, radix puerariae, Wanglaoji herbal tea, batch number: 20140901 (specifications 310ml/ bottle);

Instrument. Gastrointestinal biological instrument: Hefei Austrian source science and Technology Development Co., Ltd. production of EGEG-2D type of gastrointestinal detection instrument.

Animals. 60 rats weighing $18 \sim 22 \mathrm{~g}$, male and female, Kunming mice were provided by Shandong Lukang animal experiment center, Certificate No. No 0023555.

\section{Experimental Methods}

Take the weight 60 of $18-22 \mathrm{~g}$ mice, male and female were randomly, divided into 5 groups, one group was the control group. The remaining 4 groups of mice drinking herbal tea herbal tea source [2]. Fusen high dose, low dose of Fusen herbal tea (high-dose diluted concentration), Wang Laoji Wang Laoji (Wang Laoji dose, small dose of high dose diluted times, concentration) will replace the corresponding herbal tea drinking water of mice; blank group were given normal drinking water. Drinking herbal tea or water (mouse enough for drinking, drinking water every day to replace the mouse) bottle contents every day, continuous $17 \mathrm{~d}$. According to the observation of mouse cage drinking water, feed weight every 5 days[3]. In 16d, mice were anesthetized with ether, in the gastric antrum surface projection of the shearing, 95\% ethanol degreasing, the disc electrode with cotton balls containing saline cover tape fixed; the left forelimb wrist as reference electrode and the left hind tarsus as a ground electrode. EGEG-2D gastrointestinal electrographic instrument were 
recorded 5min electrogastrogram (egg). To observe the effect on gastrointestinal motility and observation index is the main frequency and amplitude. Until the mice, to wake up and back into the cages, fasting $(12 \mathrm{~h}$ ) water but and 17 cervical dislocation mice were killed and the stomach and duodenum, cut along the greater curvature of the stomach, the contents full with saline wash, macroscopic observation.

\section{Experimental Results}

\section{Effect Of Herbal Tea On Mice Fusen Source Weight}

Table 1 Effect of Fu Senyuan on mice body weight of herbal tea $(\bar{x} \pm \mathrm{s})$

\begin{tabular}{cccccc}
\hline GROUP & $\mathrm{n}$ & $1 \mathrm{st}$ & 6 th & 11 th & 16 th \\
\hline Blank control group & 12 & $23.03 \pm 2.06$ & $26.23 \pm 2.64$ & $30.11 \pm 4.40$ & $32.18 \pm 5.05$ \\
High dose Fusen tea group & 12 & $21.63 \pm 1.65$ & $25.8 \pm 2.50$ & $26.15 \pm 2.82 * *$ & $27.09 \pm 1.39 *$ \\
Small dose Fusen tea group & 12 & $21.13 \pm 1.45$ & $24.14 \pm 3.42$ & $25.24 \pm 3.82^{* *}$ & $26.80 \pm 2.47 *$ \\
The large dose Wanglaoji group & 12 & $19.78 \pm 1.71$ & $23.03 \pm 2.39^{* *}$ & $23.11 \pm 3.12^{* *}$ & $23.53 \pm 2.90^{* *}$ \\
The small dose Wanglaoji group & 12 & $20.5 \pm 0.71$ & $23.09 \pm 1.97 * *$ & $24.6 \pm 2.95^{* *}$ & $25.14 \pm 2.679 * *$ \\
\hline
\end{tabular}

* indicates that the blank group is better than $P<0.01$, * says that the blank group is better than the $P<0.05$

From the table we can see that changes in body weight of mice after drinking water, in the $1 \mathrm{~d}$ group and blank control group had no significant difference $(P>0.05)$, that group uniform; at $6 \mathrm{~d}$, large and small dose group significantly reduced body weight $(P<0.01)$, large, small dose of herbal tea on mice body weight Fusen little change; in 12d, large and small dose group and small Fusen herbal tea, dose group were significantly Wanlaoji herb tea weight loss $(P<0.01) ; 17 \mathrm{~d}$, large and small dose group can make Fusen herbal tea body weight of mice decreased significantly $(P<0.05)$, small dose of Wanglaoji herbal tea set can make the body weight of mice significantly decreased $(P$ $<0.01)$. The results suggest that the effect of herbal tea on weight less than Fusen and suggests that drinking a lot of Wanglaoji; short-term Fusen has little effect on tea, body weight in mice.But a large number of long-term drinking Fusen herbal tea can be slightly affected, the mice weight of feed, water consumption is basically the same.

\section{Fusen Source Herbal Tea On Mice Feed, The Effect Of Water Consumption}

Table 2 Fusen source herbal tea on mice feed, the effect of water consumption (per cage 12) (volume/ml)

\begin{tabular}{cccccc}
\hline & $\begin{array}{c}\text { Blank control } \\
\text { group }\end{array}$ & $\begin{array}{c}\text { High dose } \\
\text { Fusen tea group }\end{array}$ & $\begin{array}{c}\text { Small dose } \\
\text { Fusen tea group }\end{array}$ & $\begin{array}{c}\text { The large dose } \\
\text { Wanglaoji } \\
\text { group }\end{array}$ & $\begin{array}{c}\text { The small dose } \\
\text { Wanglaoji } \\
\text { group. }\end{array}$ \\
\hline $\begin{array}{c}\text { Feed average }(\mathrm{g}) \\
\begin{array}{c}\text { Drinking average } \\
\text { water(mL) }\end{array}\end{array}$ & 53.025 & 48.80625 & 48.3625 & 45.69375 & 46.66875 \\
\hline
\end{tabular}

From the table we can see that the change in the amount of feed of drinking herbal tea after the mice, from the beginning of $1 \mathrm{~d}$ until $17 \mathrm{~d}$, the average value can be found, large, small dose group mice feed Fusen herbal tea are basically the same, large, small dose group feed Wang Laoji herbal tea are basically the same. On the whole, small dose of Fusen tea group mice feed slightly more than large, small doses of Wang Laoji herbal tea. Changes in the amount of water drinking tea after the mice, from the beginning of $1 \mathrm{~d}$, until $17 \mathrm{~d}$ the average value can be found in high dose group mice for drinking herbal tea's demand for slightly more than the low dose group of small dose of Wang Laoji's herbal tea; herbal tea mice to the drinking water demand is slightly more than the high dose group of the whole Wang Laoji herbal tea; large and small dose group of mice drinking herbal tea's demand is more than big, small dose of Wang Laoji group. 


\section{Effect Of Herbal Tea On Mice Fusen Source Gastrointestinal Bioelectricity}

Table 3 Effect of herbal tea on mice Fusen source gastrointestinal bioelectricity $(\bar{x} \pm \mathrm{s})$

\begin{tabular}{cccc} 
Group & $N$ & Frequency(min) & Amplitude(Mv) \\
\hline Blank control group & 12 & $3.36 \pm 0.31$ & $123.7 \pm 18.38$ \\
High dose Fusen tea group & 12 & $3.25 \pm 0.29$ & $112.99 \pm 15.66$ \\
Small dose Fusen tea group & 12 & $3.24 \pm 0.35$ & $112.15 \pm 16.56$ \\
The large dose Wanglaoji group & 12 & $3.17 \pm 0.31^{*}$ & $107.70 \pm 16.09$ \\
The small dose Wanglaoji group & 12 & $3.18 \pm 0.32 *$ & $108.14 \pm 13.77$ \\
\hline
\end{tabular}

Note: compared with the blank control group, $* P<0.05$

The basic function of gastrointestinal drink herbal tea a reduction of the amplitude of Wanglaoji and herbal tea herbal tea of Wanglaoji herbal tea from the table can be seen, and the blank control group, and small dose Fusen group although the mouse gastrointestinal bioelectric frequency slightly decreased, but no significant difference; large, small dose group mice gastrointestinal bioelectric frequency slightly decreased significantly $(P<0.05)$. Large and small doses of Fusen group, small dose of Wanglaoji herbal tea group, although the mice biological gastric electrical amplitude slightly reduced, but there was no significant difference; large and small dose groups of mice gastrointestinal bioelectric effects than large, small dose Fusen group slightly larger, suggesting that a large number of long-term Fusen herbal tea in normal mice had no significant adverse effects.

\section{Summary}

Gastrointestinal motor function is an important part of the physiological function of the digestive tract. The dysfunction is the cause of gastrointestinal symptoms in one of the decisive factors [5]. for gastrointestinal motor function most can express is gastrointestinal bioelectric expression. Normal people use a lot of cool traditional Chinese medicine may lead to gastrointestinal function disorder, gastrointestinal $\mathrm{Xu}$ chaos will lead to people eating and elimination of abnormal, [6] is often accompanied by insomnia, anxiety, attention and lax wait for a phenomenon, hence the observed long-term drinking a lot of Fusen herbal tea on the body weight of normal mice, drinking water and feed amount and biological changes of whether there is the influence.

Experiments show that the change of body weight of mice after drinking water, in the $1 \mathrm{~d}$ group and blank control group had no significant difference $(P>0.05)$, that group uniform; at $6 \mathrm{~d}$, large and small dose group significantly reduced body weight $(P<0.01)$, large, small doses of Fusun herbal tea on mice body weight changes in 12d; when large, small dose group and small Fusen herbal tea, dose group were significantly Wanlaoji herb tea weight loss $(P<0.01)$; 17d, large and small dose group can make herbal tea Fusen body weight of mice decreased significantly $(P<0.05)$, low dose group of Wanglaoji herbal tea can make the mice weight decreased significantly $(P<0.01)$. It is suggested that herbal tea has little effect on the body weight of mice Fusun, but a large number of long-term drinking Fusen herbal tea can be a slight impact on body weight in mice, and the feed consumption of drinking water are basically the same. Large and small dose of herbal tea group although the mice gastrointestinal Fusen bioelectrical frequency decreased slightly, but there is no tomorrow Significant difference; that a large number of long-term drinking herbal tea on the gastrointestinal function of normal mice's basic no obvious adverse effects.

Although Fusen source of herbal tea in cold traditional Chinese medicine [7], but according to the weight of the normal mice, drinking water and feed amount and the digestive function of the situation. It is proved that the Fusen herbal tea for the normal mice produced no obvious adverse 
effects. At the same time, this experiment provides the theoretical and experimental basis for the production of Fusen herbal tea.

\section{References}

[1] L.Yu.,X.W.Zhang.,G.C.Zhao.,Y.Wang, Study on efficacy and safety of herbal tea drink tea. 25(2010)914-916.

[2] C.Li., C.J.Wang., Comparison of gastric mucosal injury in 3 kinds of spleen deficiency syndrome model rats. Chinese Journal of integrated traditional Chinese and Western Medicine. 21(2013)127-129.

[3] F.X.Wu.,Y.Hong.,N.Hai,Effect of rhubarb on plasma somatostatin and gastric mucosal injury in rats with spleen qi deficiency.National Medical Forum.25(2010)35-37.

[4]W.L.Lu.,Z.Q.Fang.,Z.Q.Pan.,X.M.Zhang and L.Hou.Different methods with different syndromes in mice induced by experimental gastric ulcer.Hunan Journal of Traditional Chinese Medicine.24(2008)70-78.

[5] L.Q.Xu., L.Qin.Study on the effect of bitter cold medicine on gastrointestinal function.Journal of Shandong University of Traditional Chinese Medicine.34(2011)554-556.

[6]M.F.Ge., W.L.Zhou., J.P.Zhang. Effect of external application of Xiaoji powder on gastrointestinal dynamics.Integrated Chinese and Western Medicine.02(2009)130-132.

[7]Y.F.Guo.,W.L.Zhou., Study on the protective effects of cuttlebone polysaccharides on gastric mucosa in mice.Academic Journal of Second Military Medical University. 25(2008)1328-1332.

[8]Q.Luo.,P.Xi.,X.Z.Liao.Experimental study of Radix Fici Hirtae Decoction on gastrointestinal motor function in mice with spleen deficiency of rhubarb.Pharmacy Today.22(2012)398-401. 